\title{
Exponential convergence of Cohen-Grossberg neural networks with continuously distributed leakage delays
}

\author{
Zhibin Chen ${ }^{1}$ and Shuhua Gong ${ }^{2 *}$
}

"Correspondence:

shuhuagong@aliyun.com

${ }^{2}$ College of Mathematics, Physics

and Information Engineering,

Jiaxing University, Jiaxing, Zhejiang

314001, P.R. China

Full list of author information is

available at the end of the article

\begin{abstract}
This paper is concerned with the global exponential convergence of Cohen-Grossberg neural networks with continuously distributed leakage delays. By using the Lyapunov functional method and differential inequality techniques, we propose a new approach to establishing some sufficient conditions ensuring that all solutions of the networks converge exponentially to the zero point. Our results complement some recent ones.
\end{abstract}

MSC: $34 \mathrm{C} 25 ; 34 \mathrm{~K} 13 ; 34 \mathrm{~K} 25$

Keywords: Cohen-Grossberg neural network; global exponential convergence; continuously distributed delay; leakage term

\section{Introduction}

It is well known that Cohen-Grossberg neural networks (CGNNs) have been successfully applied in many fields such as pattern recognition, parallel computing, associative memory, and combinatorial optimization (see [1-5]). Such applications heavily depend on the global exponential convergence behaviors, because the exponential convergent rate can be unveiled. Many good results on the problem of the global exponential convergence of the equilibriums and periodic solutions of for CGNNs are given in the literature. We refer the reader to [6-13] and the references cited therein. Recently, in real applications, a typical time delay called leakage (or 'forgetting') delay has been introduced in the negative feedback terms of the neural network system, and these terms are variously known as forgetting or leakage terms (see [14-16]). Subsequently, Gopalsamy [17] investigated the stability on the equilibrium for the bidirectional associative memory (BAM) neural networks with constant delay in the leakage term. Following this, the authors of [18-22] dealt with the existence and stability of equilibrium and periodic solutions for neuron networks model involving constant leakage delays. In particular, Peng [23] established some delay dependent criteria for the existence and global attractive periodic solutions of the bidirectional associative memory neural network with continuously distributed delays in the leakage terms. However, to the best of our knowledge, few authors have considered the exponential convergence behavior for all solutions of CGNNs with continuously distributed delays in the leakage terms. Motivated by the arguments above, in the present paper, we shall consider the following CGNNs with time-varying coefficients and continuously dis-

C2014 Chen and Gong; licensee Springer. This is an Open Access article distributed under the terms of the Creative Commons Attribution License (http://creativecommons.org/licenses/by/2.0), which permits unrestricted use, distribution, and reproduction in any medium, provided the original work is properly cited. 
tributed delays in the leakage terms:

$$
\begin{aligned}
x_{i}^{\prime}(t)= & -a_{i}\left(t, x_{i}(t)\right)\left[b_{i}\left(t, \int_{0}^{\infty} \delta_{i}(s) x_{i}(t-s) d s\right)-\sum_{j=1}^{n} c_{i j}(t) f_{j}\left(x_{j}\left(t-\tau_{i j}(t)\right)\right)\right. \\
& \left.-\sum_{j=1}^{n} d_{i j}(t) \int_{0}^{\infty} K_{i j}(u) g_{j}\left(x_{j}(t-u)\right) d u+I_{i}(t)\right], \quad i=1,2, \ldots, n,
\end{aligned}
$$

where $a_{i}$ and $b_{i}$ are continuous functions on $R^{2}, \delta_{i}, \tau_{i j}, f_{j}, g_{j}, c_{i j}, d_{i j}$ and $I_{i}$ are continuous functions on $R ; n$ corresponds to the number of units in a neural network; $x_{i}(t)$ denotes the potential (or voltage) of cell $i$ at time $t ; a_{i}$ represents an amplification function; $b_{i}$ is an appropriately behaved function; $c_{i j}(t)$ and $d_{i j}(t)$ denote the strengths of connectivity between cell $i$ and $j$ at time $t$, respectively; the activation functions $f_{i}(\cdot)$ and $g_{i}(\cdot)$ show how the $i$ th neuron reacts to the input, $\tau_{i j}(t) \geq 0$ corresponds to the transmission delays, $K_{i j}(u)$ and $\delta_{i}(u) \geq 0$ correspond to the transmission delay kernels, and $I_{i}(t)$ denotes the $i$ th component of an external input source introduced from outside the network to cell $i$ at time $t$ for $i, j \in F=\{1,2, \ldots, n\}$.

Throughout this paper, for $i, j \in F$, it will be assumed that $h_{i}:[0,+\infty) \rightarrow[0,+\infty)$ and $K_{i j}:[0,+\infty) \rightarrow R$ are continuous functions, and there exist constants $\tau_{i j}^{+}, \overline{I_{i}}, \overline{c_{i j}}$, and $\overline{d_{i j}}$ such that

$$
\tau_{i j}^{+}=\sup _{t \in R} \tau_{i j}(t), \quad \overline{I_{i}}=\sup _{t \in R}\left|I_{i}(t)\right|, \quad \overline{c_{i j}}=\sup _{t \in R}\left|c_{i j}(t)\right|, \quad \overline{d_{i j}}=\sup _{t \in R}\left|d_{i j}(t)\right| .
$$

We also make the following assumptions.

$\left(\mathrm{H}_{1}\right)$ For each $j \in F$, there exist nonnegative constants $\beta, \alpha, \tilde{L}_{j}$ and $L_{j}$ such that

$$
0 \leq \beta \leq 1,0 \leq \alpha \leq 1, \quad\left|f_{j}(u)\right| \leq \tilde{L}_{j}|u|^{\beta}, \quad\left|g_{j}(u)\right| \leq L_{j}|u|^{\alpha}
$$

for all $u \in R$.

$\left(\mathrm{H}_{2}\right)$ For $i \in F$, there exist positive constants $\underline{a_{i}}$ and $\overline{a_{i}}$ such that

$$
\underline{a_{i}} \leq a_{i}(t, u) \leq \overline{a_{i}} \text { for all } t>0, u \in R
$$

$\left(\mathrm{H}_{3}\right)$ For $i \in F, b_{i}(t, 0) \equiv 0$, and there exist positive constants $\underline{b_{i}}$ and $\overline{b_{i}}$ such that

$$
\underline{b_{i}}|u-v| \leq \operatorname{sgn}(u-v)\left(b_{i}(t, u)-b_{i}(t, v)\right) \leq \overline{b_{i}}|u-v| \quad \text { for all } t>0, u, v \in R .
$$

$\left(\mathrm{H}_{4}\right)$ For all $t>0$ and $i, j \in F$, there exist constants $\eta>0$ and $\lambda>0$ such that

$$
\int_{0}^{\infty} s \delta_{i}(s) e^{\lambda s} d s<+\infty, \quad \int_{0}^{\infty}\left|K_{i j}(u)\right| e^{\lambda u} d u<+\infty
$$

and

$$
\begin{aligned}
-\eta> & -\left[\underline{a_{i}} \underline{b_{i}} \int_{0}^{\infty} \delta_{i}(s) e^{\lambda s} d s-\lambda\left(1+\overline{a_{i}} \overline{b_{i}} \int_{0}^{\infty} s \delta_{i}(s) e^{\lambda s} d s\right)\right. \\
& \left.-\overline{a_{i}} \overline{b_{i}} \int_{0}^{\infty} s \delta_{i}(s) e^{\lambda s} d s \overline{a_{i}} \overline{b_{i}} \int_{0}^{\infty} \delta_{i}(s) e^{\lambda s} d s\right]
\end{aligned}
$$




$$
\begin{aligned}
& +\overline{a_{i}}\left[\sum_{j=1}^{n} \tilde{L}_{j}\left(\left|c_{i j}(t)\right| e^{\lambda \beta \tau_{i j}(t)}+\overline{a_{i}} \overline{b_{i}} \int_{0}^{\infty} s \delta_{i}(s) e^{\lambda s} d s \overline{c_{i j}} e^{\lambda \beta \tau_{i j}^{+}}\right) e^{\lambda(1-\beta) t}\right. \\
& \left.+\sum_{j=1}^{n} L_{j} \int_{0}^{\infty}\left|K_{i j}(u)\right| e^{\lambda \alpha u} d u\left(\left|d_{i j}(t)\right|+\overline{d_{i j}} \overline{a_{i}} \overline{b_{i}} \int_{0}^{\infty} s \delta_{i}(s) e^{\lambda s} d s\right) e^{\lambda(1-\alpha) t}\right]
\end{aligned}
$$

$\left(\mathrm{H}_{5}\right) I_{i}(t)=O\left(e^{-\lambda t}\right)(t \rightarrow \pm \infty), i \in F$.

The initial conditions associated with system (1.1) are of the form

$$
x_{i}(s)=\varphi_{i}(s), \quad s \in(-\infty, 0], i \in F,
$$

where $\varphi_{i}(\cdot)$ denotes a real-valued bounded continuous function defined on $(-\infty, 0]$.

The remaining part of this paper is organized as follows. In Section 2, we present some new sufficient conditions to ensure that all solutions of CGNNs (1.1) with initial conditions (1.4) converge exponentially to the zero point. In Section 3, we shall give some examples and remarks to illustrate our results obtained in the previous sections.

\section{Main results}

Theorem 2.1 Let $\left(\mathrm{H}_{1}\right)-\left(\mathrm{H}_{5}\right)$ hold. Then, for every solution $Z(t)=\left(x_{1}(t), x_{2}(t), \ldots, x_{n}(t)\right)^{T}$ of CGNNs (1.1) with initial conditions (1.4), there exists a positive constant $K$ such that

$$
\left|x_{i}(t)\right| \leq K e^{-\lambda t} \quad \text { for all } t>0, i \in F \text {. }
$$

Proof Let $Z(t)=\left(x_{1}(t), x_{2}(t), \ldots, x_{n}(t)\right)^{T}$ be a solution of system (1.1) with initial conditions (1.4), and let

$$
X_{i}(t)=e^{\lambda t} x_{i}(t), \quad i \in F
$$

In view of (1.1), we have

$$
\begin{aligned}
X_{i}^{\prime}(t)= & \lambda X_{i}(t)+e^{\lambda t} a_{i}\left(t, x_{i}(t)\right)\left[-b_{i}\left(t, \int_{0}^{\infty} \delta_{i}(s) e^{\lambda(s-t)} X_{i}(t-s) d s\right)\right. \\
& \left.+\sum_{j=1}^{n} c_{i j}(t) f_{j}\left(x_{j}\left(t-\tau_{i j}(t)\right)\right)+\sum_{j=1}^{n} d_{i j}(t) \int_{0}^{\infty} K_{i j}(u) g_{j}\left(x_{j}(t-u)\right) d u-I_{i}(t)\right] \\
= & \lambda X_{i}(t)+e^{\lambda t} a_{i}\left(t, x_{i}(t)\right)\left[-b_{i}\left(t, \int_{0}^{\infty} \delta_{i}(s) e^{\lambda(s-t)} X_{i}(t) d s\right)\right. \\
& +\left(b_{i}\left(t, \int_{0}^{\infty} \delta_{i}(s) e^{\lambda(s-t)} X_{i}(t) d s\right)-b_{i}\left(t, \int_{0}^{\infty} \delta_{i}(s) e^{\lambda(s-t)} X_{i}(t-s) d s\right)\right) \\
& +\sum_{j=1}^{n} c_{i j}(t) f_{j}\left(x_{j}\left(t-\tau_{i j}(t)\right)\right) \\
& \left.+\sum_{j=1}^{n} d_{i j}(t) \int_{0}^{\infty} K_{i j}(u) g_{j}\left(x_{j}(t-u)\right) d u-I_{i}(t)\right], \quad i=1,2, \ldots, n .
\end{aligned}
$$


Let

$$
M=\max _{i=1,2, \ldots, n} \sup _{s \leq 0}\left\{e^{\lambda s}\left|\varphi_{i}(s)\right|\right\} .
$$

From (1.2) and $\left(\mathrm{H}_{5}\right)$, we can choose a positive constant $K>M+1$ such that

$$
\eta>\frac{\left[1+\overline{a_{i}} \overline{b_{i}} \int_{0}^{\infty} s \delta_{i}(s) e^{\lambda s} d s\right] \overline{a_{i}} \sup _{t \in R}\left|e^{\lambda t} I_{i}(t)\right|}{K} \text { for all } t>0, i \in F
$$

Then it is easy to see that

$$
\left|X_{i}(t)\right| \leq M<K \quad \text { for all } t \leq 0, i=1,2, \ldots, n \text {. }
$$

We now claim that

$$
\left|X_{i}(t)\right|<K \quad \text { for all } t>0, i \in F \text {. }
$$

Otherwise, one of the following two cases must occur.

(1) There exist $i \in F$ and $t^{*}>0$ such that

$$
X_{i}\left(t^{*}\right)=K, \quad\left|X_{j}(t)\right|<K \quad \text { for all } t<t^{*}, j \in F .
$$

(2) There exist $i \in F$ and $t^{* *}>0$ such that

$$
X_{i}\left(t^{* *}\right)=-K, \quad\left|X_{j}(t)\right|<K \quad \text { for all } t<t^{* *}, j \in F .
$$

Now, we distinguish two cases to finish the proof.

Case (1). If (2.5) holds. Then, from (2.1), (2.3), and $\left(\mathrm{H}_{1}\right)-\left(\mathrm{H}_{4}\right)$, we have

$$
\begin{aligned}
0 \leq & X_{i}^{\prime}\left(t^{*}\right) \\
= & \lambda X_{i}\left(t^{*}\right)+e^{\lambda t^{*}} a_{i}\left(t^{*}, x_{i}\left(t^{*}\right)\right)\left[-b_{i}\left(t^{*}, \int_{0}^{\infty} \delta_{i}(s) e^{\lambda\left(s-t^{*}\right)} X_{i}\left(t^{*}\right) d s\right)\right. \\
& +\left(b_{i}\left(t^{*}, \int_{0}^{\infty} \delta_{i}(s) e^{\lambda\left(s-t^{*}\right)} X_{i}\left(t^{*}\right) d s\right)-b_{i}\left(t^{*}, \int_{0}^{\infty} \delta_{i}(s) e^{\lambda\left(s-t^{*}\right)} X_{i}\left(t^{*}-s\right) d s\right)\right) \\
& +\sum_{j=1}^{n} c_{i j}\left(t^{*}\right) f_{j}\left(x_{j}\left(t^{*}-\tau_{i j}\left(t^{*}\right)\right)\right) \\
& \left.+\sum_{j=1}^{n} d_{i j}\left(t^{*}\right) \int_{0}^{\infty} K_{i j}(u) g_{j}\left(x_{j}\left(t^{*}-u\right)\right) d u-I_{i}\left(t^{*}\right)\right] \\
\leq & \lambda X_{i}\left(t^{*}\right)-\underline{a_{i}} \underline{b}_{i} \int_{0}^{\infty} \delta_{i}(s) e^{\lambda s} d s X_{i}\left(t^{*}\right)+\overline{a_{i}} \overline{b_{i}} \int_{0}^{\infty} \delta_{i}(s) e^{\lambda s} \int_{t^{*}-s}^{t^{*}} X_{i}^{\prime}(u) d u d s \\
& +\overline{a_{i}} \sum_{j=1}^{n}\left|c_{i j}\left(t^{*}\right)\right| \tilde{L}_{j} e^{\lambda \beta \tau_{i j}\left(t^{*}\right)} e^{\lambda(1-\beta) t^{*}}\left|X_{j}\left(t^{*}-\tau_{i j}\left(t^{*}\right)\right)\right|^{\beta} \\
& +\overline{a_{i}} \sum_{j=1}^{n}\left|d_{i j}\left(t^{*}\right)\right| L_{j} e^{\lambda(1-\alpha) t^{*}} \int_{0}^{\infty}\left|K_{i j}(u)\right| e^{\lambda \alpha u}\left|X_{j}\left(t^{*}-u\right)\right|^{\alpha} d u+\overline{a_{i}} e^{\lambda t^{*}}\left|I_{i}\left(t^{*}\right)\right|
\end{aligned}
$$


Chen and Gong Journal of Inequalities and Applications 2014, 2014:48

Page 5 of 8

http://www.journalofinequalitiesandapplications.com/content/2014/1/48

$$
\begin{aligned}
& \leq \lambda X_{i}\left(t^{*}\right)-\underline{a_{i}} \underline{b_{i}} \int_{0}^{\infty} \delta_{i}(s) e^{\lambda s} d s X_{i}\left(t^{*}\right)+\overline{a_{i}} \overline{b_{i}} \int_{0}^{\infty} \delta_{i}(s) e^{\lambda s} \int_{t^{*}-s}^{t^{*}} \mid \lambda X_{i}(u) \\
& +e^{\lambda u} a_{i}\left(u, x_{i}(u)\right)\left[-b_{i}\left(u, \int_{0}^{\infty} \delta_{i}(v) e^{\lambda(v-u)} X_{i}(u-v) d v\right)+\sum_{j=1}^{n} c_{i j}(u) f_{j}\left(x_{j}\left(u-\tau_{i j}(u)\right)\right)\right. \\
& \left.+\sum_{j=1}^{n} d_{i j}(u) \int_{0}^{\infty} K_{i j}(v) g_{j}\left(x_{j}(u-v)\right) d v-I_{i}(u)\right] \mid d u d s \\
& +\overline{a_{i}} \sum_{j=1}^{n}\left|c_{i j}\left(t^{*}\right)\right| \tilde{L}_{j} e^{\lambda \beta \tau_{i j}\left(t^{*}\right)} e^{\lambda(1-\beta) t^{*}}\left|X_{j}\left(t^{*}-\tau_{i j}\left(t^{*}\right)\right)\right|^{\beta} \\
& +\overline{a_{i}} \sum_{j=1}^{n}\left|d_{i j}\left(t^{*}\right)\right| L_{j} e^{\lambda(1-\alpha) t^{*}} \int_{0}^{\infty}\left|K_{i j}(u)\right| e^{\lambda \alpha u}\left|X_{j}\left(t^{*}-u\right)\right|^{\alpha} d u+\overline{a_{i}} e^{\lambda t^{*}}\left|I_{i}\left(t^{*}\right)\right| \\
& \leq \lambda X_{i}\left(t^{*}\right)-\underline{a_{i}} \underline{b_{i}} \int_{0}^{\infty} \delta_{i}(s) e^{\lambda s} d s X_{i}\left(t^{*}\right)+\lambda \overline{a_{i}} \overline{b_{i}} \int_{0}^{\infty} s \delta_{i}(s) e^{\lambda s} d s X_{i}\left(t^{*}\right) \\
& +\overline{a_{i}} \overline{b_{i}} \int_{0}^{\infty} s \delta_{i}(s) e^{\lambda s} d s \overline{a_{i}} \overline{b_{i}} \int_{0}^{\infty} \delta_{i}(s) e^{\lambda s} d s X_{i}\left(t^{*}\right) \\
& +\overline{a_{i}} \bar{b}_{i} \int_{0}^{\infty} \delta_{i}(s) e^{\lambda s} \int_{t^{*}-s}^{t^{*}} \overline{a_{i}}\left[\sum_{j=1}^{n} \bar{c}_{i j} \tilde{L}_{j} e^{\lambda \beta \tau_{i j}^{+}} e^{\lambda(1-\beta) u}\left|X_{j}\left(u-\tau_{i j}(u)\right)\right|^{\beta}\right. \\
& \left.+\sum_{j=1}^{n} \overline{d_{i j}} L_{j} e^{\lambda(1-\alpha) u} \int_{0}^{\infty}\left|K_{i j}(v)\right| e^{\lambda \alpha v}\left|X_{j}(u-v)\right|^{\alpha} d v+\sup _{t \in R}\left|e^{\lambda t} I_{i}(t)\right|\right] d u d s \\
& +\overline{a_{i}} \sum_{j=1}^{n}\left|c_{i j}\left(t^{*}\right)\right| \tilde{L}_{j} e^{\lambda \beta \tau_{i j}\left(t^{*}\right)} e^{\lambda(1-\beta) t^{*}}\left|X_{j}\left(t^{*}-\tau_{i j}\left(t^{*}\right)\right)\right|^{\beta} \\
& +\overline{a_{i}} \sum_{j=1}^{n}\left|d_{i j}\left(t^{*}\right)\right| L_{j} e^{\lambda(1-\alpha) t^{*}} \int_{0}^{\infty}\left|K_{i j}(u)\right| e^{\lambda \alpha u}\left|X_{j}\left(t^{*}-u\right)\right|^{\alpha} d u+\overline{a_{i}} e^{\lambda t^{*}}\left|I_{i}\left(t^{*}\right)\right| \\
& \leq-\left[\underline{a_{i}} \frac{b_{i}}{\int_{0}^{\infty}} \delta_{i}(s) e^{\lambda s} d s-\lambda\left(1+\overline{a_{i}} \overline{b_{i}} \int_{0}^{\infty} s \delta_{i}(s) e^{\lambda s} d s\right)\right. \\
& \left.-\overline{a_{i}} \overline{b_{i}} \int_{0}^{\infty} s \delta_{i}(s) e^{\lambda s} d s \overline{a_{i}} \overline{b_{i}} \int_{0}^{\infty} \delta_{i}(s) e^{\lambda s} d s\right] X_{i}\left(t^{*}\right) \\
& +\overline{a_{i}}\left[\sum_{j=1}^{n} \tilde{L}_{j}\left(\left|c_{i j}\left(t^{*}\right)\right| e^{\lambda \beta \tau_{i j}\left(t^{*}\right)}+\overline{a_{i}} \overline{b_{i}} \int_{0}^{\infty} s \delta_{i}(s) e^{\lambda s} d s \overline{c_{i j}} e^{\lambda \beta \tau_{i j}^{+}}\right) e^{\lambda(1-\beta) t^{*}}\right. \\
& \left.+\sum_{j=1}^{n} L_{j} \int_{0}^{\infty}\left|K_{i j}(u)\right| e^{\lambda \alpha u} d u\left(\left|d_{i j}\left(t^{*}\right)\right|+\overline{d_{i j}} \overline{a_{i}} \overline{b_{i}} \int_{0}^{\infty} s \delta_{i}(s) e^{\lambda s} d s\right) e^{\lambda(1-\alpha) t^{*}}\right] K \\
& +\left[1+\overline{a_{i}} \overline{b_{i}} \int_{0}^{\infty} s \delta_{i}(s) e^{\lambda s} d s\right] \overline{a_{i}} \sup _{t \in R}\left|e^{\lambda t} I_{i}(t)\right| \\
& =\left\{-\left[\underline{a_{i}} \underline{b_{i}} \int_{0}^{\infty} \delta_{i}(s) e^{\lambda s} d s-\lambda\left(1+\overline{a_{i}} \overline{b_{i}} \int_{0}^{\infty} s \delta_{i}(s) e^{\lambda s} d s\right)\right.\right. \\
& \left.-\overline{a_{i}} \overline{b_{i}} \int_{0}^{\infty} s \delta_{i}(s) e^{\lambda s} d s \overline{a_{i}} \overline{b_{i}} \int_{0}^{\infty} \delta_{i}(s) e^{\lambda s} d s\right] \\
& +\overline{a_{i}}\left[\sum_{j=1}^{n} \tilde{L}_{j}\left(\left|c_{i j}\left(t^{*}\right)\right| e^{\lambda \beta \tau_{i j}\left(t^{*}\right)}+\bar{a}_{i} \overline{b_{i}} \int_{0}^{\infty} s \delta_{i}(s) e^{\lambda s} d s \overline{c_{i j}} e^{\lambda \beta \tau_{i j}^{+}}\right) e^{\lambda(1-\beta) t^{*}}\right.
\end{aligned}
$$




$$
\begin{aligned}
& \left.\left.+\sum_{j=1}^{n} L_{j} \int_{0}^{\infty}\left|K_{i j}(u)\right| e^{\lambda \alpha u} d u\left(\left|d_{i j}\left(t^{*}\right)\right|+\overline{d_{i j}} \overline{a_{i}} \overline{b_{i}} \int_{0}^{\infty} s \delta_{i}(s) e^{\lambda s} d s\right) e^{\lambda(1-\alpha) t^{*}}\right]\right\} \\
& +\left[1+\overline{a_{i}} \overline{b_{i}} \int_{0}^{\infty} s \delta_{i}(s) e^{\lambda s} d s\right] \overline{a_{i}} \sup _{t \in R}\left|e^{\lambda t} I_{i}(t)\right| \\
& <-\eta K+\left[1+\overline{a_{i}} \overline{b_{i}} \int_{0}^{\infty} s \delta_{i}(s) e^{\lambda s} d s\right] \overline{a_{i}} \sup _{t \in R}\left|e^{\lambda t} I_{i}(t)\right| \\
& <0 .
\end{aligned}
$$

This contradiction implies that (2.5) does not hold.

Case (2). If (2.6) holds, then, from (2.1), (2.3), and $\left(\mathrm{H}_{1}\right)-\left(\mathrm{H}_{4}\right)$, by using a similar argument as in Case (1), we can derive a contradiction, which shows that (2.6) does not hold.

Therefore, (2.4) is proved and

$$
\left|x_{i}(t)\right| \leq K e^{-\lambda t} \quad \text { for all } t>0, i \in F
$$

This implies that the proof of Theorem 2.1 is now completed.

\section{An example}

Example 3.1 Consider the following CGNNs with time-varying delays in the leakage terms:

$$
\left\{\begin{aligned}
x_{1}^{\prime}(t)= & -\left(2+e^{\cos ^{2} t} \frac{1}{10 \pi} \arctan x_{1}(t)\right)\left[\left(4-\frac{|t||\sin t|}{1+2|t|}\right) \int_{0}^{\infty} \delta_{1}(s) x_{1}(t-s) d s\right. \\
& +\frac{1}{70} \frac{|t| \sin t}{1+40|t|} f_{1}\left(x_{1}\left(t-2 \sin ^{2} t\right)\right)+\frac{1}{70} \frac{|t| \sin t}{1+36|t|} \\
& \cdot f_{2}\left(x_{2}\left(t-3 \sin ^{2} t\right)\right)+\frac{1}{70} \frac{|t| \sin t}{1+40|t|} \int_{0}^{\infty} e^{-u} g_{1}\left(x_{j}(t-u)\right) d u \\
& \left.+\frac{1}{70} \frac{|t|^{2} \sin t}{1+36|t|^{2}} \int_{0}^{\infty} e^{-u} g_{2}\left(x_{j}(t-u)\right) d u+20,000 e^{-3 t} \sin t\right] \\
x_{2}^{\prime}(t)= & -\left(2+e^{\sin ^{2} t} \frac{1}{10 \pi} \arctan x_{2}(t)\right)\left[\left(4-\frac{|t||\cos t|}{1+2|t|}\right) \int_{0}^{\infty} \delta_{2}(s) x_{2}(t-s) d s\right. \\
& +\frac{1}{70} \frac{|t| \cos t}{1+40|t|} f_{1}\left(x_{1}\left(t-2 \sin ^{2} t\right)\right) \\
& +\frac{1}{70} \frac{|t| \cos t}{1+36|t|} f_{2}\left(x_{2}\left(t-5 \sin ^{2} t\right)\right)+\frac{1}{70} \frac{|t| \cos t}{1+40|t|} \int_{0}^{\infty} e^{-u} g_{1}\left(x_{j}(t-u)\right) d u \\
& \left.+\frac{1}{70} \frac{|t| \cos t}{1+36|t|} \int_{0}^{\infty} e^{-u} g_{2}\left(x_{j}(t-u)\right) d u+30,000 e^{-t} \cos t\right]
\end{aligned}\right.
$$

where $f_{i}(x)=g_{i}(x)=x \sin ^{2 i} x, \delta_{i}(t)=e^{-10 t}, i=1,2$.

It follows that

$$
1 \leq \underline{a_{i}} \leq \overline{a_{i}} \leq 3, \quad 3 \leq \underline{b_{i}} \leq \overline{b_{i}} \leq 4, \quad i=1,2
$$

and

$$
\underline{b_{i}}|u| \leq \operatorname{sgn}(u) b_{i}(t, u) \quad \text { for all } t, u \in R, i=1,2 \text {. }
$$

Define a continuous function $\Gamma_{i}(\omega)$ by setting

$$
\begin{aligned}
\Gamma_{i}(\omega)= & -\left[\underline{a_{i}} \underline{b_{i}} \int_{0}^{\infty} \delta_{i}(s) e^{\omega s} d s-\omega\left(1+\overline{a_{i}} \overline{b_{i}} \int_{0}^{\infty} s \delta_{i}(s) e^{\omega s} d s\right)\right. \\
& \left.-\overline{a_{i}} \overline{b_{i}} \int_{0}^{\infty} s \delta_{i}(s) e^{\omega s} d s \overline{a_{i}} \overline{b_{i}} \int_{0}^{\infty} \delta_{i}(s) e^{\omega s} d s\right]+\overline{a_{i}}\left[\sum _ { j = 1 } ^ { n } \tilde { L } _ { j } \left(\left|c_{i j}(t)\right| e^{\omega \beta \tau_{i j}(t)}\right.\right.
\end{aligned}
$$




$$
\begin{aligned}
& \left.+\overline{a_{i}} \overline{b_{i}} \int_{0}^{\infty} s \delta_{i}(s) e^{\omega s} d s \overline{c_{i j}} e^{\omega \beta \tau_{i j}^{+}}\right) e^{\omega(1-\beta) t}+\sum_{j=1}^{n} L_{j} \int_{0}^{\infty}\left|K_{i j}(u)\right| e^{\omega \alpha u} d u\left(\left|d_{i j}(t)\right|\right. \\
& \left.\left.+\overline{d_{i j}} \overline{a_{i}} \overline{b_{i}} \int_{0}^{\infty} s \delta_{i}(s) e^{\omega s} d s\right) e^{\omega(1-\alpha) t}\right] \quad \text { for all } t>0, i=1,2 .
\end{aligned}
$$

According to the continuity of $\Gamma_{i}(\omega)$ and $\Gamma_{i}(0)<0$, we can choose constants $\eta=0.1$ and $\lambda>0$ such that

$$
\Gamma_{i}(\lambda)<-\eta \quad \text { for all } t>0, i=1,2,
$$

which implies that the CGNNs (3.1) satisfied $\left(\mathrm{H}_{1}\right)-\left(\mathrm{H}_{5}\right)$. Hence, from Theorem 2.1, all solutions of the CGNNs (3.1) with initial value $\left(\varphi_{1}(x), \varphi_{2}(x)\right)$ converge exponentially to the zero point $(0,0)$.

Remark 3.1 It is easy to check that the results in [17-23] and [24-34] are invalid for the global exponential convergence of (3.1), since the leakage delays are continuously distributed.

\section{Competing interests}

The authors declare that they have no competing interests.

\section{Authors' contributions}

ZC gave the proof of Theorem 2.1 and drafted the manuscript. SG proved and gave the example to illustrate the effectiveness of the obtained results. All authors read and approved the final manuscript.

\section{Author details}

${ }^{1}$ School of Science, Hunan University of Technology, Zhuzhou, Hunan 412000, P.R. China. ${ }^{2}$ College of Mathematics, Physics and Information Engineering, Jiaxing University, Jiaxing, Zhejiang 314001, P.R. China.

\section{Acknowledgements}

The authors would like to express the sincere appreciation to the reviewers for their helpful comments in improving the presentation and quality of the paper. This work was supported by the National Natural Science Foundation of China (grant nos. 51375160, 11201184), and the Scientific Research Fund of Hunan Provincial Natural Science Foundation of China (grant no. 12JJ3007).

Received: 4 April 2013 Accepted: 7 January 2014 Published: 30 Jan 2014

\section{References}

1. Cohen, M, Grossberg, S: Absolute stability and global pattern formation and parallel memory storage by competitive neural networks. IEEE Trans. Syst. Man Cybern. 13, 815-826 (1983)

2. Kennedy, MP, Chua, LO: Neural networks for nonlinear programming. IEEE Trans. Circuits Syst. 35, 554-562 (1988)

3. Cao, J, Liang, J: Boundedness and stability for Cohen-Grossberg neural networks with time-varying delays. J. Math. Anal. Appl. 296, 665-685 (2004)

4. Chen, T, Rong, L: Delay-independent stability analysis of Cohen-Grossberg neural networks. Phys. Lett. A 317, 436-439 (2003)

5. Li, Y: Existence and stability of periodic solutions for Cohen-Grossberg neural networks with multiple delays. Chaos Solitons Fractals 20, 459-466 (2004)

6. Liao, X, Li, C, Wong, K: Criteria for exponential stability of Cohen-Grossberg neural networks. Neural Netw. 17, 1401-1406 (2004)

7. Wang, L: Stability of Cohen-Grossberg neural networks with distributed delays. Appl. Math. Comput. 160, 93-110 (2005)

8. Wang, L, Zou, X: Exponential stability of Cohen-Grossberg neural networks. Neural Netw. 15, 415-422 (2002)

9. Liu, B: New convergence behavior of solutions to Cohen-Grossberg neural networks with delays and time-varying coefficients. Phys. Lett. A 372(2), 117-123 (2008)

10. Gong, S: Anti-periodic solutions for a class of Cohen-Grossberg neural networks. Comput. Math. Appl. 58(2), 341-347 (2009)

11. Zhang, Z, Zhou, D: Global robust exponential stability for second-order Cohen-Grossberg neural networks with multiple delays. Neurocomputing 73(1-3), 213-218 (2009)

12. Zhang, ZQ, Yang, Y, Huang, YS: Global exponential stability of interval general BAM neural networks with reaction-diffusion terms and multiple time-varying delays. Neural Netw. 24, 457-465 (2011) 
13. Zhang, Z, Liu, W, Zhou, D: Existence and global exponential stability of periodic solution to Cohen-Grossberg BAM neural networks with time-varying delays. Abstr. Appl. Anal. 2012, Article ID 805846 (2012). doi:10.1155/2012/805846

14. Haykin, S: Neural Networks. Prentice Hall, New York (1994)

15. Kosok, B: Neural Networks and Fuzzy Systems. Prentice Hall, New Delhi (1992)

16. Gopalsamy, K: Stability and Oscillations in Delay Differential Equations of Population Dynamics. Kluwer Academic, Dordrecht (1992)

17. Gopalsamy, K: Leakage delays in BAM. J. Math. Anal. Appl. 325, 1117-1132 (2007)

18. Li, X, Cao, J: Delay-dependent stability of neural networks of neutral type with time delay in the leakage term. Nonlinearity 23, 1709-1726 (2010)

19. Li, X, Rakkiyappan, R, Balasubramaniam, P: Existence and global stability analysis of equilibrium of fuzzy cellular neural networks with time delay in the leakage term under impulsive perturbations. J. Franklin Inst. 348, 135-155 (2011)

20. Balasubramaniam, P, Vembarasan, V, Rakkiyappan, R: Leakage delays in T-S fuzzy cellular neural networks. Neural Process. Lett. 33, 111-136 (2011)

21. Liu, B: Global exponential stability for BAM neural networks with time-varying delays in the leakage terms. Nonlinear Anal., Real World Appl. (2012). doi:10.1016/j.nonrwa.2012.07.016

22. Gan, Q, Liang, Y: Synchronization of chaotic neural networks with time delay in the leakage term and parametric uncertainties based on sampled-data control. J. Franklin Inst. 349(6), 1955-1971 (2012)

23. Peng, S: Global attractive periodic solutions of BAM neural networks with continuously distributed delays in the leakage terms. Nonlinear Anal., Real World Appl. 11, 2141-2151 (2010)

24. Liu, B: Global exponential stability for BAM neural networks with time-varying delays in the leakage terms. Nonlinear Anal., Real World Appl. 14, 559-566 (2013)

25. Chen, Z, Yang, M: Exponential convergence for HRNNs with continuously distributed delays in the leakage terms. Neural Comput. Appl. (2012). doi:10.1007/s00521-012-1172-2

26. Chen, Z, Meng, J: Exponential convergence for cellular neural networks with time-varying delays in the leakage terms. Abstr. Appl. Anal. 2012, Article ID 941063 (2012). doi:10.1155/2012/941063

27. Xiong, W, Meng, J: Exponential convergence for cellular neural networks with continuously distributed delays in the leakage terms. Electron. J. Qual. Theory Differ. Equ. 2013, 10 (2013). http://www.math.u-szeged.hu/ejqtde/

28. $\mathrm{Xu}, \mathrm{Y}$ : Anti-periodic solutions for HCNNs with time-varying delays in the leakage terms. Neural Comput. Appl. (2012). doi:10.1007/s00521-012-1330-6

29. Chen, Z: A shunting inhibitory cellular neural network with leakage delays and continuously distributed delays of neutral type. Neural Comput. Appl. (2012). doi:10.1007/s00521-012-1200-2

30. Chen, Z, Meng, J: Exponential convergence for cellular neural networks with time-varying delays in the leakage terms. Abstr. Appl. Anal. 2012, Article ID 941063 (2012). doi:10.1155/2012/941063

31. Zhang, H, Yang, M: Global exponential stability of almost periodic solutions for SICNNs with continuously distributed leakage delays. Abstr. Appl. Anal. 2013, Article ID 307981 (2013)

32. Lakshmanan, S, Park, JH, Lee, TH, Jung, HY, Rakkiyappan, R: Stability criteria for BAM neural networks with leakage delays and probabilistic time-varying delays. Appl. Math. Comput. 219(17), 9408-9423 (2013)

33. Li, Y, Li, Y: Existence and exponential stability of almost periodic solution for neutral delay BAM neural networks with time-varying delays in leakage terms. J. Franklin Inst. 350(9), 2808-2825 (2013)

34. Baštinec, J, Diblík, J, Khusainov, DY, Ryvolová, A: Exponential stability and estimation of solutions of linear differential systems of neutral type with constant coefficients. Bound. Value Probl. 2010, Article ID 956121 (2010)

10.1186/1029-242X-2014-48

Cite this article as: Chen and Gong: Exponential convergence of Cohen-Grossberg neural networks with continuously distributed leakage delays. Journal of Inequalities and Applications 2014, 2014:48

\section{Submit your manuscript to a SpringerOpen ${ }^{\circ}$ journal and benefit from:}

- Convenient online submission

- Rigorous peer review

- Immediate publication on acceptance

- Open access: articles freely available online

- High visibility within the field

- Retaining the copyright to your article 